Document downloaded from:

http://hdl.handle.net/10251/64599

This paper must be cited as:

Moreno Cano, R.; Page Del Pozo, AF.; Riera Guasp, J.; Hueso Pagoaga, JL. (2015). Experimental study of viscous friction in undergraduate physics laboratory: introduction of phase diagrams to analyse dynamic equilibrium. European Journal of Physics. 36:1-15. doi:10.1088/0143-0807/36/3/035033.

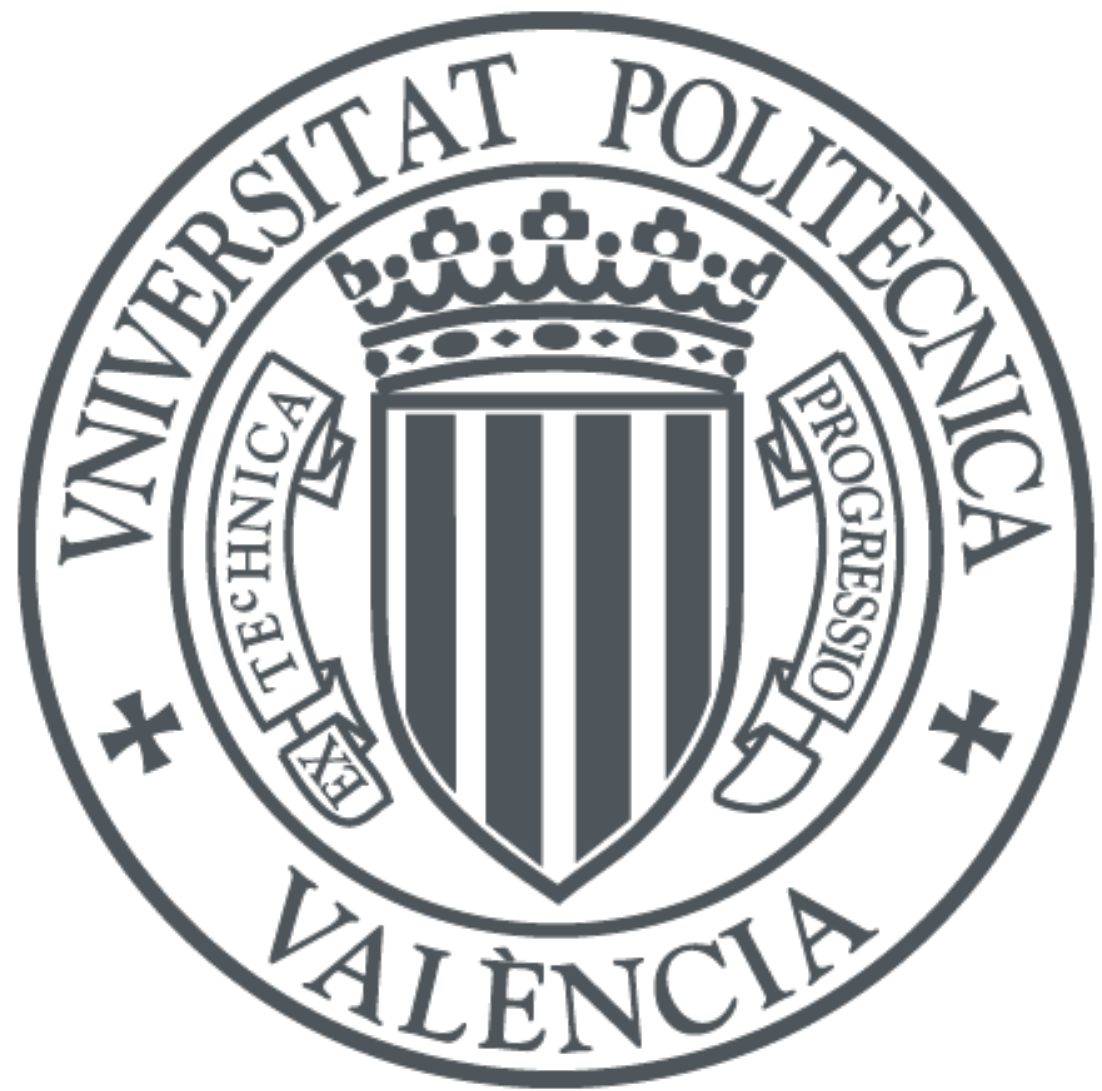

The final publication is available at

http://dx.doi.org/10.1088/0143-0807/36/3/035033

Copyright European Physical Society

Additional Information 


\title{
Experimental study of viscous friction in undergraduate physics laboratory. Introduction of phase diagrams to analyze dynamic equilibrium
}

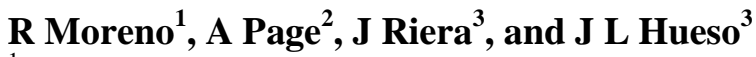 \\ ${ }^{1}$ Departamento de Física Aplicada. Universitat Politècnica de València \\ ${ }^{2}$ Instituto de Biomecánica de Valencia. Universitat Politècnica de València \\ ${ }^{3}$ Instituto de Matemática Multidisciplinar, Universitat Politècnica de València, \\ E-46071 Valencia, Spain \\ E-mail: jriera@fis.upv.es
}

\begin{abstract}
.
In this work we propose using phase diagrams for explaining the dynamical behavior of simple mechanical systems. First the motion of the system $x(t)$ is experimentally measured and then, the derivatives, $v(t)$ and $a(t)$, are obtained from it and the motion equation $f(x, v, a)=0$ is represented graphically. This idea is applied to the study of a system with linear viscous drag, explaining the evolution of the system towards the dynamical equilibrium point corresponding to the limit velocity. The phase diagrams of the viscous drag are compared with those of the Coulomb drag, which is not continuous and does not necessarily lead to a uniformly accelerated motion.The method is illustrated by an experiment in a dynamic track with magnetic damping. The use of phase diagrams allows checking the linearity of this damping. Moreover it allows identifying the existence of a small Coulomb drag between the track and the cart that appears as a small discontinuity of the function $a(v)$ when the direction of the movement changes.
\end{abstract}

PACS numbers: 89.20.Ff

Submitted to: Eur. J. Phys.

\section{Introduction}

Friction is an important part in the study of mechanics in the first year university. In high school, the Coulomb drag is studied in the context of constant forces and uniformly accelerated motions [1, 2].

The study of viscous friction is introduced in first year university courses. The simplest case is the linear friction $F_{r}=-K v$ that is applied to the analysis of problems like the fall of a sphere in a fluid in laminar regime (Stokes law) or the magnetic damping or the study of damped oscillations. Another kind of friction is the quadratic friction associated to the movement of objects inside a fluid in turbulent regime, such as the drag force in the air.

The analysis of problems with viscous friction has a great docent interest, because it allows introducing important concepts such as the dynamical equilibrium and the limit velocity. In this 
line, several experiences oriented to the study of the air drag in falling objects have been proposed [3-6].

Despite its pedagogical interest, this kind of experiences presents some difficulties, both at the experimental and the methodological levels. From the experimental point of view, it is difficult to produce repeatable movements when working with light objects subject to the air drag. Even under very controlled conditions, the onset of turbulences or of the Magnus effect [7] may alter the measurement conditions quite. Besides, the dependence on the velocity of the viscous drag is not constant, and even may not follow a simple model. In fact, in turbulent regime, the relation force-velocity is statistic, and replicating the same experiment in the same conditions may produce different movements, which can be detected by measuring with enough precision $[8,9]$.

Magnetic damping is a good alternative for producing repeatable linear friction. If one puts magnets in a cart on an aluminum dynamical track, the Eddy currents associated to the flux variation generate a viscous friction of linear type and turbulence free, $F_{r}=-K v$, tending to compensate the flux variation (Lenz law).

Besides the experimental drawbacks, one also must consider some methodological limitations. In most of the examined works, the dynamical analysis stands on stating Newton's second law and integrating the differential equation for obtaining the movement equation, $x(t)$, which is tested against the experimental results. This procedure presents two important shortcomings. On one hand, it does not make much sense assuming a given dependence of the friction force when the objective is just determining a model for that force. This is especially important in the viscous friction case, where, in many instances, the model is not clear or even can vary along the motion [10]. Furthermore, as shown in [11], very different differential equations can give as result similar movement equations.

On the other hand, in order to obtain the equation of the trajectory, one has to integrate differential equations that, sometimes, exceed the knowledge of first year students. For example, certain movements such as the parabolic shot with friction do not have explicit analytic solution and it must be obtained numerically [12-14].

An alternative that avoids these limitations consists in checking the differential equation of the motion with the direct measurement of position, velocity and acceleration. This strategy has been used in former works by using photogrammetry and efficient techniques of numerical differentiation for estimating the values of the position and its derivatives [11, 15].

In this way, one can introduce in the first year syllabus some tools of great interest, such as phase diagrams. Traditionally, the phase diagrams have been frequently used in the study of nonlinear dynamics in different fields: economics [16], biological systems evolution [17], as much as in many specific physical phenomena such as non-linear coupled oscillations [18] or the non-linear dynamics of a pendulum [19]. In general, these kinds of diagrams are interesting 
for the study of systems with complex dynamics, because they allow analyzing their evolution from expressions of the form $f(x, v, a)=0$ without having to integrate any differential equation. Nevertheless, these diagrams have been scarcely used in the undergraduate physics laboratory, possibly due to the difficulty of measuring directly and with enough precision the involved variables.

In this line, the objective of the present work is to present a laboratory experiment for the first year of physics consisting in the analysis of mechanical systems subject to viscous friction by using phase diagrams. This procedure allows easily identifying the dependence of the friction force on the velocity, as much as deepening in important concepts such as limit velocity and dynamical equilibrium. For the experimental estimation of the relevant variables of the process, we propose a video photogrammetry system and advanced techniques of numerical differentiation [15]. This measurement system is adequate for the level of the undergraduate physics laboratory and has been utilized in former works for analyzing complex movements, obtaining satisfactory results [11].

The use of the measurement technique alongside the phase diagrams allows detecting the coexistence of Coulomb and viscous frictions in the same experiment. This is difficult to observe from the fit of $x(t)$ so that it has not been considered in other works on the same experiment that used conventional measurement techniques.

\section{Material and Methods}

\subsection{Theoretical frame}

The phase diagram is a graphic showing the relation between the different variables that define the dynamics of a movement, that is to say, relations of the form $f(x, v, a)=0$. A point of the curve corresponds to a dynamical state of the system at a given time instant and the temporal evolution of the system is obtained moving along the curve. The phase diagrams allow qualitative studies of the differential equation without needing to solve it and provide insight into important characteristics such as dynamical equilibrium, periodicity or stability [20]. In summary, they are an interesting tool for analyzing complex problems in first year university courses, allowing the students to focus on the dynamical details instead of on solving an involved mathematical problem.

Now, we explain the $a(v)$ diagrams in different situations where linear viscous or Coulomb frictions appear in order to interpret the results of the experiment of movement with friction.

\subsubsection{Linear viscous friction.}

Figure 1 shows the forces scheme and the phase diagram $a(v)$ for the cases of a particle subject to linear viscous friction (a.1) and (a.2), respectively, and for the particle subject to linear 
viscous friction plus a constant force, (b.1) and (b.2). From these diagrams it is easy to interpret the dynamics of the movement. Namely, the movement of a mobile launched with initial velocity on a horizontal plane with viscous friction (a.1) corresponds to segment $\boldsymbol{B L}$ in the phase diagram (a.2). The particle has positive speed and negative acceleration, and so the velocity decreases until reaching the equilibrium point, $a=0$, corresponding in this case to $v=0$. If the particle is launched with negative velocity (towards the left), the movement corresponds to segment $\boldsymbol{C L}$ in the phase diagram.

Figure 1.b.1 shows the case where besides the friction force, there is a constant force, like the weight component in an inclined plane $F_{W} \sin \alpha$. Releasing the mobile with initial speed zero, the motion corresponds to segment $\boldsymbol{a}_{\mathbf{0}} \boldsymbol{L}$, which starts with velocity zero and positive initial acceleration. As the velocity increases, the friction makes the acceleration to decrease until reaching the dynamical equilibrium at point $\boldsymbol{L}$ where $a=0$ and $v=v_{\text {lim }}$. The starting point $\boldsymbol{B}$ means launching the mobile downwards with velocity greater than the limit velocity. On the contrary, to start from $\boldsymbol{C}$ implies launching the particle upwards (negative $v$ ). The particle climbs with decreasing velocity until stopping and then descends with increasing velocity tending to $v_{\text {lim }}$.

(a.1)

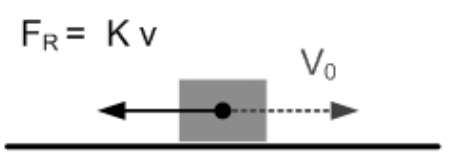

(b.1)

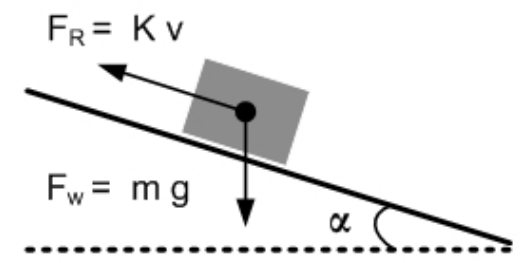

(a.2)

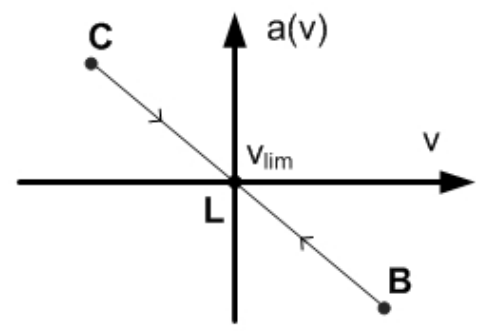

(b.2)

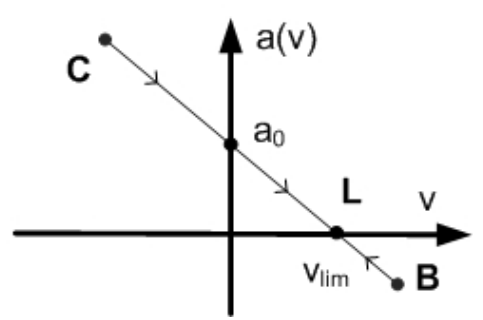

Figure 1. Examples of movements with linear viscous friction. Forces scheme (a.1) and phase plane (a.2) of the motion in the horizontal plane. Forces scheme (b.1) and phase plane (b.2) of the motion in the inclined plane.

If the model were not linear, instead of the line, we would have another curve, but always with negative slope at $v=0$ (or null, depending on the model). The negative slope condition is 
a characteristic of the dissipative forces that guarantees the existence of a limit velocity. Actually, if the intersection of $a(v)$ with the $v$-axis has negative slope, then, departing from $\boldsymbol{L}$, the acceleration has opposite sign to the velocity and tends to return to this dynamical equilibrium point. A positive slope means that the particle accelerates if $v>v_{\text {lim }}$ or decelerates if $v<v_{\text {lim }}$, so that $v=v_{\text {lim }}$ is an unstable equilibrium point.

Traditionally, this problem is solved by integrating the equation of the motion

$$
m g \sin \alpha-K v=m \frac{d v}{d t}
$$

which allows obtaining $v(t)$ and $x(t)$.

$$
\begin{gathered}
v(t)=v_{\lim }\left(1-e^{-\frac{K}{m} t}\right)+v_{o} e^{-\frac{K}{m} t} \\
x(t)=x_{o}+\frac{m}{K}\left(v_{o}-v_{T}\right)\left(1-e^{-\frac{K}{m} t}\right)+v_{l i m} t
\end{gathered}
$$

where $x_{0}$ and $v_{0}$ are the initial conditions for position and velocity. As the coefficients of the exponents show, the speed of convergence to the limit depends on $K / \mathrm{m}$.

Moreover, the limit velocity $v_{\text {lim }}$, given by (4) is obtained by imposing the equilibrium condition $\frac{d v}{d t}=0$ on (1) and solving for $v$. In the horizontal plane case (figure 1 a.1) the limit velocity is 0 .

$$
v_{\text {lim }}=\frac{m g \sin \alpha}{K}
$$

The experimental check of equation (3) is not a simple task for first year students, because they must know how to integrate the equation (this is easy in the linear case, but more difficult for quadratic friction or frictions of other type). Moreover, the fit of $x(t)$ to the experimental data is nonlinear.

\subsubsection{Coulomb friction.}

Figure 2 represents two configurations of a mobile gliding on a plane under the action of Coulomb friction. Scheme (a.1) corresponds to a body launched with positive initial velocity $v_{0}$ on a horizontal plane. The trajectory in the phase plane (a.2) is $\boldsymbol{B} \boldsymbol{a}_{\mathbf{0}+}$. The constant negative acceleration lasts until the body stops. If the initial velocity is negative, the acceleration is positive describing the trajectory $\boldsymbol{C} \boldsymbol{a}_{\mathbf{0}}$ in the phase plane. In both cases, the limit velocity is null. 
(a.1)

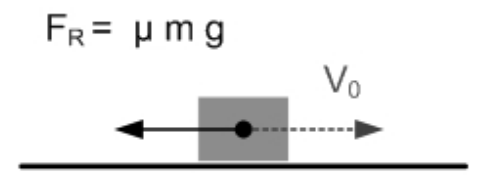

(b.1)

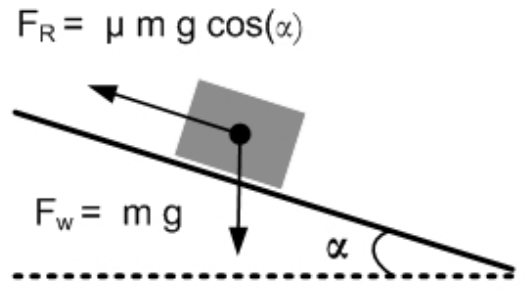

(a.2)

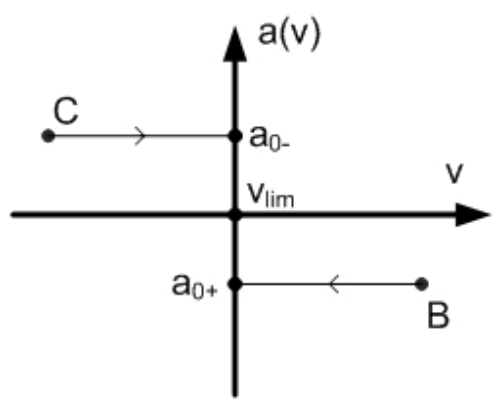

(b.2)

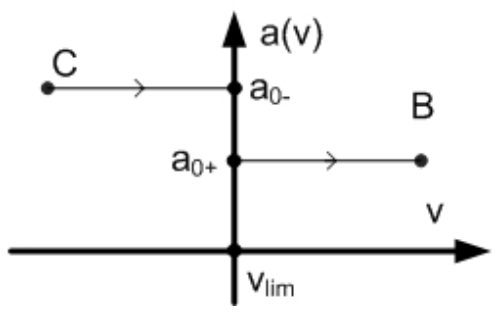

Figure 2. Examples of movements with Coulomb friction. Forces scheme (a.1) and phase plane (a.2) of the motion in the horizontal plane. Forces scheme (b.1) and phase plane (b.2) of the motion in the inclined plane.

Diagrams b of figure 2 correspond to a similar setting, but now the motion takes place in the inclined plane. In this case, besides the friction force, we have to consider the term $m g \sin \alpha$. The result is a diagram similar to the former one, but displaced upwards $a_{1}=g \sin \alpha$. The onset of Coulomb friction complicates quite the analytic resolution of the problems of dynamics due to the discontinuity at $v=0$, that prevents having a unique analytic expression for the whole range of the movement. For example, in the case of the inclined plane, assuming $\tan \alpha>\mu$, one has the following equation

$$
m g \sin \alpha-\operatorname{sign}(v) \mu m g \cos \alpha=m a
$$

Observe that, for $v_{0}<0$, there is a positive acceleration $a_{0 \text { - }}$ that makes the (negative) velocity increase to 0 . From this point on, the body is subject to a different acceleration $a_{0+}$ that increases $v$ further. Then, it is not a true uniformly accelerated movement because there are two different accelerations. This is an important detail that is generally omitted and the inclined plane is presented as a typical example of uniformly accelerated movement. In fact the students think that Coulomb friction and constant acceleration come together.

In the simplest case, for $v_{0}>0$, the sign of the velocity does not change and one gets the classic solution of a uniformly accelerated movement according to the equations:

$$
\begin{gathered}
v(t)=v_{0}+g[\sin \alpha-\mu \cos \alpha] t \\
x(t)=x_{0}+v_{0} t+\frac{1}{2} g[\sin \alpha-\mu \cos \alpha] t^{2}
\end{gathered}
$$




\subsubsection{Viscous and Coulomb friction.}

We finally consider the general case of a movement subject simultaneously to viscous and Coulomb friction. Figure 3 shows the free body diagrams and the trajectories in the phase plane for two cases: body launched on a horizontal plane and launched on an inclined plane.

(a.1)

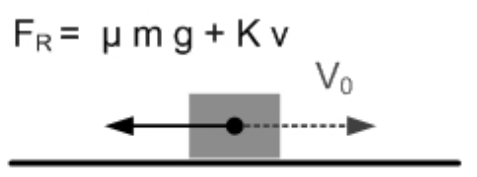

(b.1)

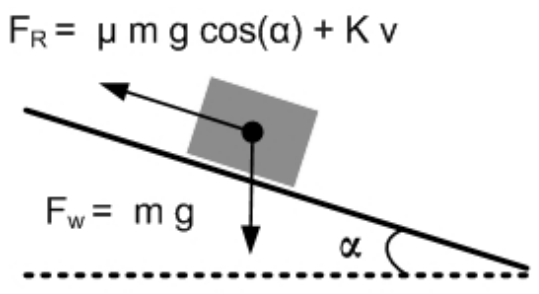

(a.2)

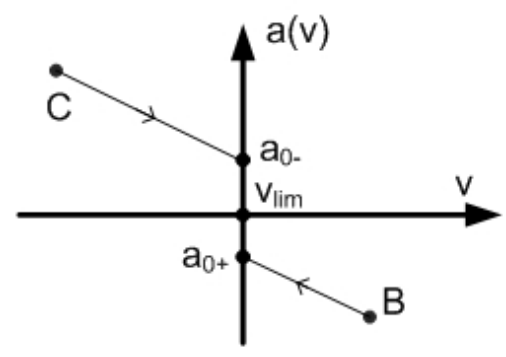

(b.2)

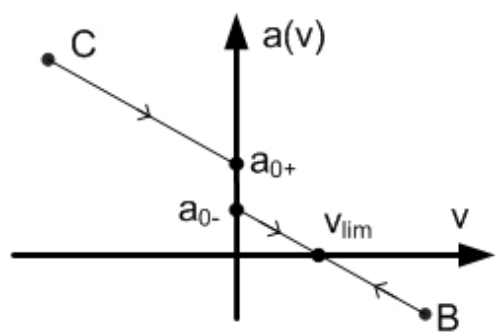

Figure 3. Examples of movements with linear viscous friction and Coulomb friction. Forces scheme (a.1) and phase plane (a.2) of the motion in the horizontal plane. Forces scheme (b.1) and phase plane (b.2) of the motion in the inclined plane.

The differential equation is

$$
m g \sin \alpha-K v-\operatorname{sign}(v) \mu m g \cos \alpha=m \frac{d v}{d t}
$$

This equation is similar to the differential equation of the viscous friction in the inclined plane (1), but modifying the ordinate at the origin in the phase plane. In fact, to the constant acceleration term $g \sin \alpha$ one has to add the term associated to Coulomb friction $\operatorname{sign}(v) \mu g \cos \alpha$, whose sign depends on the sign of the velocity and that introduces a discontinuity at $v=0$. The limit velocity is also different, including a term due to Coulomb friction,

$$
v_{\text {lim }}=\frac{m g \sin \alpha-\operatorname{sign}(v) \mu m g \cos \alpha}{K}
$$

It is important to point out that Coulomb friction introduces a discontinuity in the differential equation when the sign of the velocity changes. When the mobile is launched 
upwards on the inclined plane, while ascending $(v<0)$ the component of the weight in the movement direction and the friction force have the same sign and produce a positive acceleration $a_{0-}=g(\sin \alpha+\mu \cos \alpha)$ that finally stops the mobile $(v=0)$. At this point, the descent begins only if the weight component in the movement direction is bigger than Coulomb friction. In that case, initial descent acceleration is $a_{0+}=g(\sin \alpha-\mu \cos \alpha)$, which produces a discontinuity in $a(v)$. As the velocity grows, due to the viscous friction the resulting acceleration decreases to zero, reaching the dynamical equilibrium point where the mobile descends at constant limit speed $v_{\text {lim }}$.

\subsection{Experimental Setup}

In order to generate a movement with linear viscous friction, we propose the experimental setup shown in figure 4. It consists in an aluminum cinematic track with variable inclination, what allows adjusting the system acceleration to values as small as desired. The $\mathrm{X}$ axis has been taken in the movement direction, taking the positive axis in the descent direction. The inclination of the track has been measured by means of a plumb.

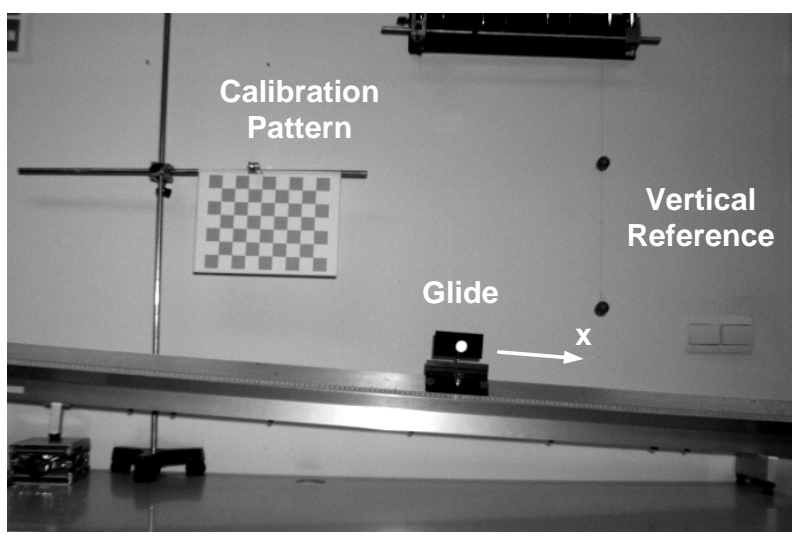

Figure 4. Experimental setup for the generation of a movement with linear viscous friction. Observe the cinematic track with the glider, the plumb to ascertain the vertical direction and the calibration pattern [23].

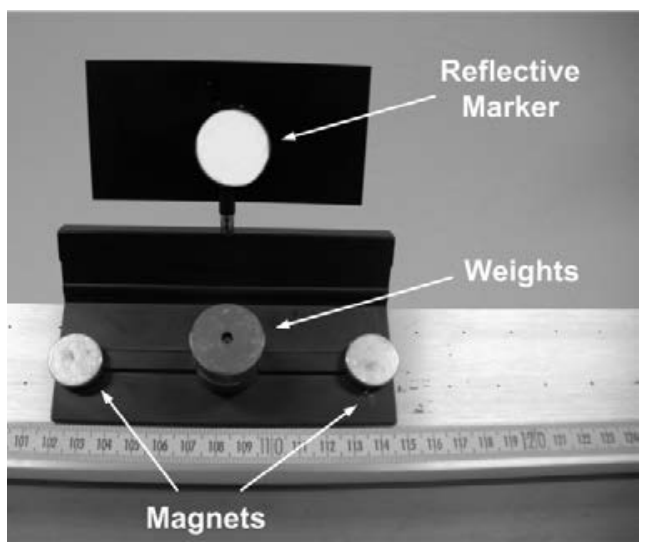

Figure 5. Glider detail. Observe the reflective marker used for the digital image tracking, the magnet and the weights located in the frontal part of the glider.

Figure 5 shows the glider in detail. Four magnets have been symmetrically located in the ends of the glider, as explained in [21, 22]. The mass of the system has been modified by placing additional weights of $100 \mathrm{~g}$.

The movement has been recorded with a digital video camera Mikrotron EoSens_CL_MC1362, with 800×600 pixel spatial resolution and frame rate $200 \mathrm{fps}$. A spherical reflective marker has been attached to the frontal part of the glider in order to facilitate the tracking of the image. The marker detection and the position measurement have been performed according to the procedure 
described in [23]. The 2D restitution technique used corrects the effects of perspective and optical distortion, so that the precision of the position measurements is $0.1 \%$.

\subsection{Analyzed movement}

We have measured the slider motion on the track for two different inclination angles: $0^{\circ}$ and $4^{\circ}$. For each inclination, we have considered different glider masses $(m 1=335 \mathrm{~g}, \mathrm{~m} 2=435 \mathrm{~g}$ and $\mathrm{m3}=535 \mathrm{~g}$ ). For each inclination-mass combination, a launch in each direction has been measured. The glider has been launched manually, and so, the initial velocities of each movement are different.

\subsection{Data processing}

The measurement system provides positions in terms of time, $x_{r}\left(t_{j}\right)$ at given time instants $t_{j}, j=1, \ldots, N$. The positions are affected by random errors, which prevent the computation of the derivatives by using finite differences. Then, for the computation of velocities and accelerations, we use the smoothing and numerical differentiation technique based on the local fit of a kernel described in [15]. This system allows the direct estimation of the first and second derivatives at each time instant without using any predefined parametric model. The procedure consists in fitting locally a third degree polynomial about each point $x_{r}\left(t_{j}\right)$, that minimizes the error function defined by

$$
S S E_{j}=\sum_{i=1}^{N} w_{i j}\left\{x_{r}\left(t_{j}\right)-\left[a_{j}\left(t_{j}-t_{i}\right)^{3}+b_{j}\left(t_{j}-t_{i}\right)^{2}+c_{j}\left(t_{j}-t_{i}\right)+d_{j}\right]\right\}^{2}
$$

where $w_{i j}$ is the weight of the $i$-th observation in the fit about $x\left(t_{j}\right)$. This weight depends on the difference $\left(t_{j}-t_{i}\right)$. Concretely, we use the following Gaussian kernel

$$
w_{i j}=(2 \pi)^{-1 / 2} \exp \left(-\frac{\left(t_{i}-t_{j}\right)^{2}}{2 h^{2}}\right)
$$

where $h$ is the bandwidth, a parameter that controls the smoothing degree. A small value of $h$ implies that only the closest neighbors to $t_{j}$ have a significant weight, whereas a big value of $h$ means that the local fit takes into account points located at certain distance of $t_{j}$. Correctly selecting the value $h$ is critical in the smoothing process. Small bandwidths produce a poor smoothing that produces sharpness in the fitted function and its derivatives. On the contrary, big bandwidths suppose an excess of smoothing that can eliminate part of the useful information. Here we have estimated the suitable value for $h$ according to the method described in [15].

Once fitted the parameters $a_{j}, b_{j}, c_{j}$ and $d_{j}$ according to (10), the values of the position $x\left(t_{j}\right)$ and its derivatives $v\left(t_{j}\right)$ and $a\left(t_{j}\right)$ can be obtained from 


$$
\begin{gathered}
x\left(t_{j}\right)=d_{j} \\
v\left(t_{j}\right)=c_{j} \\
a\left(t_{j}\right)=2 b_{j}
\end{gathered}
$$

The resulting velocities and accelerations for each mass configuration have been represented

in an $a-v$ diagram and a linear fit of type $a(v)=-\frac{K}{m} v+a_{0}$ has been computed. The fit has been performed separately for the zones $v<0$ and $v>0$, which has permitted identifying discontinuities at $v=0$ and consequently, the existence of Coulomb friction. The goodness of the fit has been measured by the squared correlation coefficient $R^{2}$. Finally, the limit velocity has been obtained as the zero intercept of $a(v)$.

\section{Results}

\subsection{Horizontal track}

In the horizontal track, we have taken 6 independent measurements, corresponding to 3 different masses and 2 launch directions.

Figure 6 shows the obtained values for $a(v)$ marked with dots and the fitted linear model in solid lines for each movement. Table 1 shows the numerical values of the linear fit. 


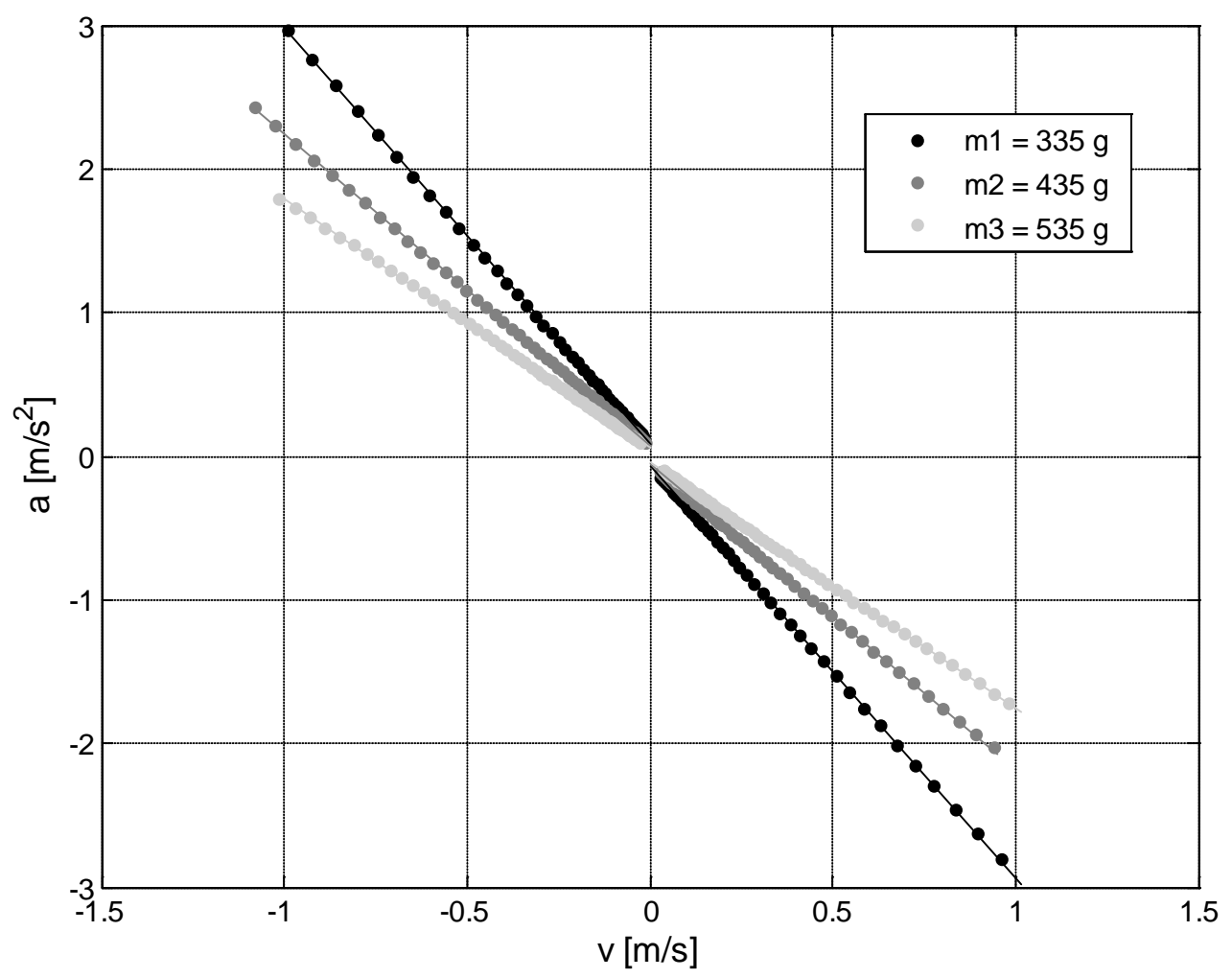

Figure 6. $a(v)$ diagrams for the horizontal track. The points correspond to the experimental values and the lines to the fits. Observe the linear dependence of the acceleration on the velocity and that the slope is lower for higher mass values.

Table 1 shows that the measured acceleration values closely follow a linear model in all the cases, with $R^{2}>0.9998$. It is also shown that the slope decreases as the glider mass increases, with similar values for both directions of the launch (differences lower than $2 \%$ ).

Table 1. Linear fit coefficients for the 3 mass configurations and 2 movement directions in the horizontal track.

\begin{tabular}{crrrl}
\hline & \multicolumn{4}{c}{$a(\mathbf{v})=-(\mathrm{K} / \mathbf{m}) \mathbf{v}+\mathbf{a}_{\mathbf{0}}$} \\
\hline Mass & & $\mathbf{a}_{\mathbf{0}}\left(\mathbf{m} / \mathbf{s}^{2}\right)$ & $\mathbf{K} / \mathbf{m}(\mathbf{1} / \mathbf{s})$ & $\mathbf{R}^{2}$ \\
\hline \multirow{2}{*}{$\mathrm{m} 1: 335 \mathrm{~g}$} & $\mathrm{v}<0$ & 0,0770 & 2,8998 & 0,9999 \\
& $\mathrm{v}>0$ & $-0,0692$ & 2,8608 & 1,0000 \\
\hline \multirow{2}{*}{$\mathrm{m} 2: 435 \mathrm{~g}$} & $\mathrm{~V}<0$ & 0,0703 & 2,1671 & 1,0000 \\
& $\mathrm{v}>0$ & $-0,0614$ & 2,1098 & 0,9999 \\
\hline \multirow{2}{*}{$\mathrm{m} 3: 535 \mathrm{~g}$} & $\mathrm{v}<0$ & 0,0548 & 1,7427 & 0,9998 \\
& $\mathrm{v}>0$ & $-0,0443$ & 1,7159 & 0,9999 \\
\hline
\end{tabular}


Observe that, in the fit, the values of the independent term $a_{0}$ are small but nonzero and that their signs change systematically with the movement direction. This result is coherent with the existence of a small Coulomb friction.

Figure 7 shows a detail of the $a-v$ observations for small velocities. According to the theoretical model, the intersection with the axis of ordinates, $v=0$, should be located at $\pm \mu g$, independently of the mass value. Nevertheless, the figure shows a dependence on the glider mass. Concretely, there is a slight increase of $\mu$ as the mass decreases.

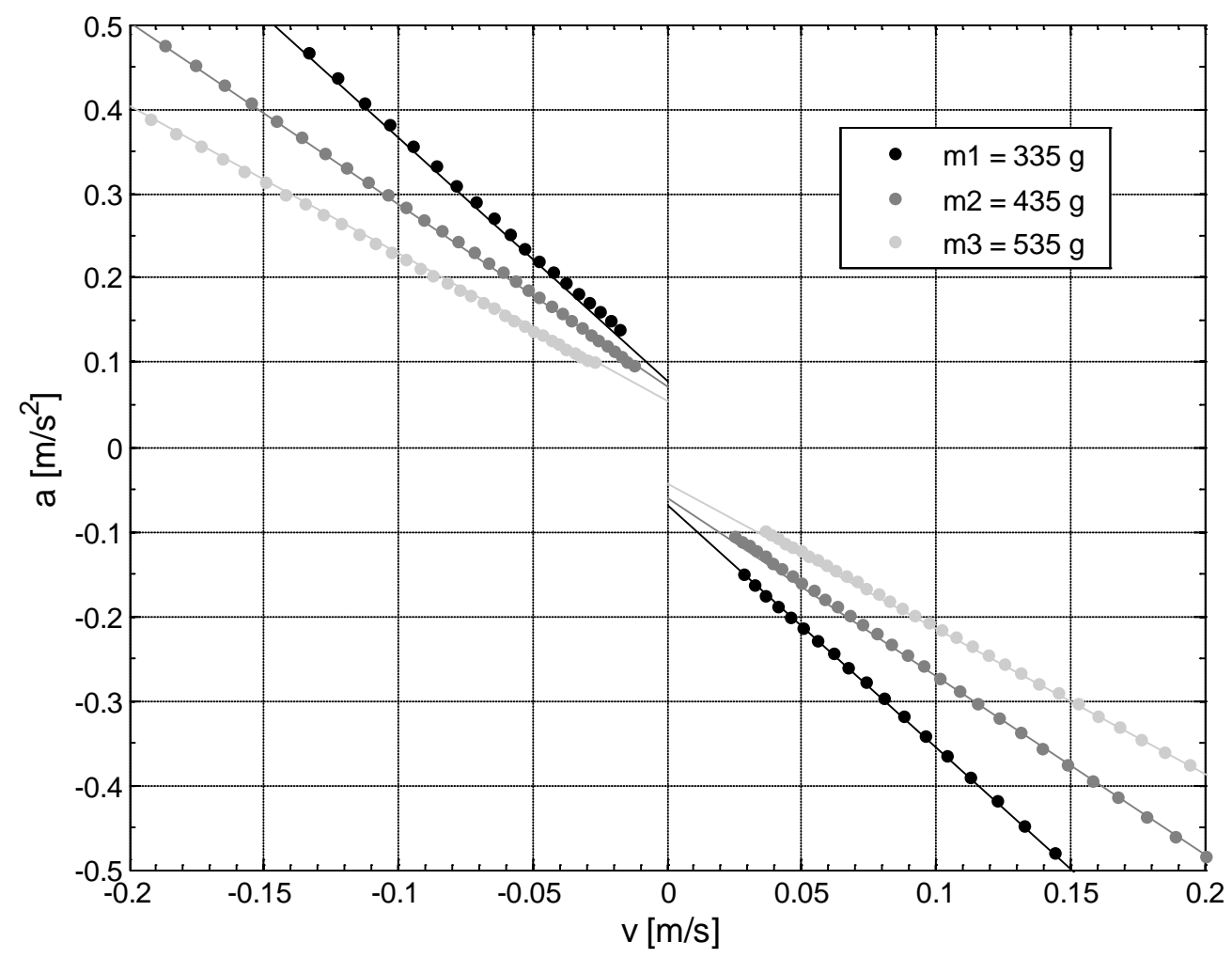

Figure 7. Detail of the zone of small velocities in the $a-v$ diagram. Observe that the fitted lines do not touch the origin, but there is a systematic deviation of the intersection with the ordinate axis depending on the mass of the glider and with different sign according to the movement direction.

The value of the friction coefficient $\mu$ and the angle $\alpha$ for each mass configuration has been estimated from the mean of the accelerations at $v=0$ and the size of the discontinuity, according to equation

$$
\frac{a_{0-}+a_{0+}}{2}=g \sin \alpha ; \frac{a_{0-}-a_{0+}}{2}=\mu g \cos \alpha
$$

The estimated coefficients are shown in Table 2. 
Table 2. Values of the inclination and the Coulomb friction coefficient in terms of the glider mass.

\begin{tabular}{lcc}
\hline & $\alpha\left[^{\circ}\right]$ & $\mu$ \\
\hline $\mathrm{m} 1: 335 \mathrm{~g}$ & 0,0229 & 0,0075 \\
$\mathrm{~m} 2: 435 \mathrm{~g}$ & 0,0261 & 0,0067 \\
$\mathrm{~m} 3: 535 \mathrm{~g}$ & 0,0307 & 0,0051 \\
\hline
\end{tabular}

It is important to point out that the estimated value of the friction coefficient is very small, $\mu<0.01$, and that, at this order of magnitude, any variation in the system configuration, such as the glider mass, can affect the experimentally obtained numerical values. Anyway, the obtained results are of the same order as the ones obtained in other works for the same configuration of the track and the glider, 0.004 in [1] and 0.007 in [2].

\subsection{Inclined track}

In this case, we have used the same configurations as in the previous subsection except for the track inclination that is now 4 degrees, which introduces a constant force due the component of the weight in the descent direction, whose values is $F_{w}=m g \sin \alpha$.

Figure 8 shows the measured values of $a(v)$ and the linear fits for the case of the inclined track.

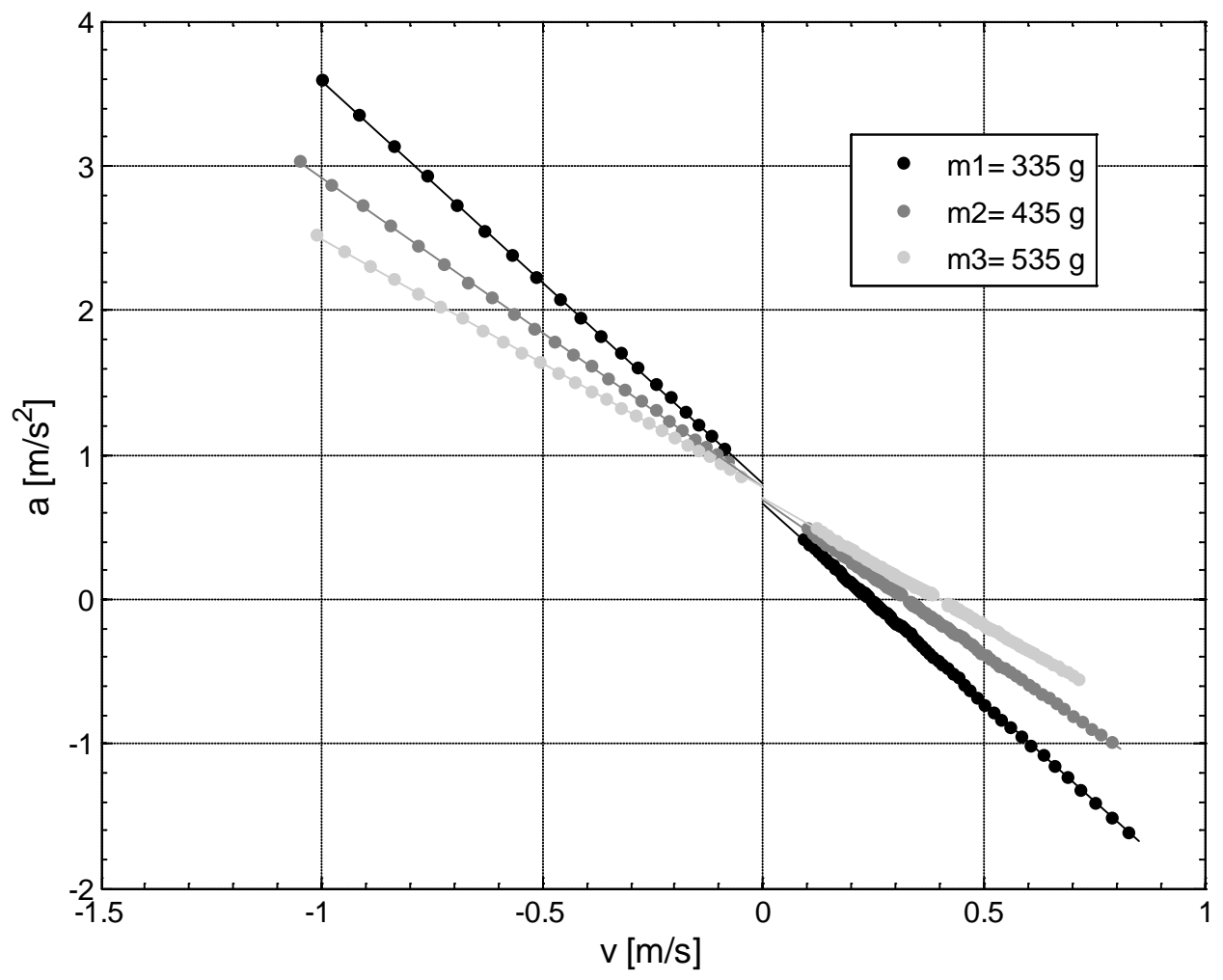


Figure 8. $a-v$ diagrams for a track with 4 degrees inclination. The experimental values are marked by points and the fits by solid lines.

Table 3 shows the fitting coefficients for the 6 different configurations of the inclined track.

Table 3. Fitting coefficients for the 6 different configurations of the inclined track.

\begin{tabular}{crrrr}
\hline & \multicolumn{4}{c}{$\mathbf{a}(\mathbf{v})=-(\mathbf{K} / \mathbf{m}) \mathbf{v}+\mathbf{a}_{\mathbf{0}}$} \\
\hline Mass & & $\mathbf{a}_{\mathbf{0}}\left(\mathbf{m} / \mathbf{s}^{\mathbf{2}}\right)$ & $\mathbf{K} / \mathbf{m} \mathbf{( 1 / s )}$ & $\mathbf{R}^{2}$ \\
\hline \multirow{2}{*}{ m1: 335 g } & $\mathbf{v}<\mathbf{0}$ & 0,8063 & 2,7792 & 0,9999 \\
& $\mathbf{v}>\mathbf{0}$ & 0,6670 & 2,7568 & 0,9998 \\
\cline { 2 - 5 } m2: 435 g & $\mathbf{v}<\mathbf{0}$ & 0,7803 & 2,1346 & 0,9999 \\
& $\mathbf{v}>\mathbf{0}$ & 0,6874 & 2,1268 & 0,9999 \\
\cline { 2 - 5 } m3: 535 g & $\mathbf{v}<\mathbf{0}$ & 0,7739 & 1,7212 & 1,0000 \\
& $\mathbf{v}>\mathbf{0}$ & 0,6950 & 1,7430 & 0,9996 \\
\hline
\end{tabular}

Notice that, in this case, the range of positive velocities is composed of two intervals corresponding to different movements. The interval $0<v<v_{\text {lim }}$ corresponds to the descent phase when the glider is launched upwards from the lower part of the track, whereas the interval $v>v_{\text {lim }}$ corresponds to launching the glider from the upper part of the track with initial velocity higher than the limit velocity. Nevertheless, the joint fit of both intervals produces good results with $R^{2}>0.9996$. In fact, the values of $K / m$ are similar to that of the horizontal track.

Figure 9 shows the detail of the $a-v$ diagram at low velocities, where the discontinuity at $v=0$ produced by Coulomb friction can be observed. With the same idea as in the former section, the track inclination and the friction coefficient have been obtained and are shown in Table 4. We can observe the same dependence of $\mu$ on the glider mass as in the case of the horizontal track. Observe also that the inclination angle for each case is very close to the value of 4 degrees initially measured by the plumb. 


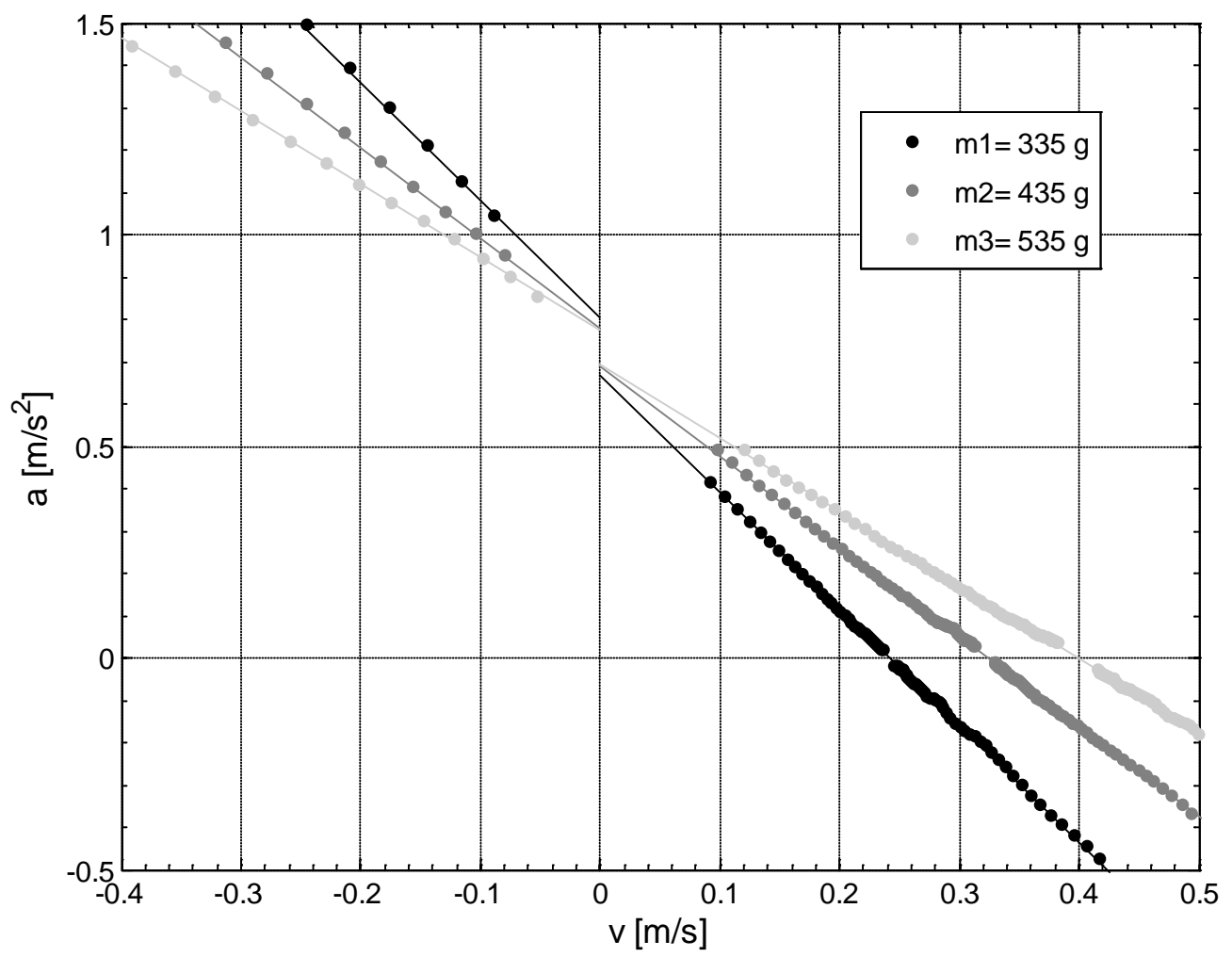

Figure 9. Detail of the $a-v$ diagram for the track inclined 4 degrees in the range of low velocities. Observe the discontinuity introduced by the Coulomb friction for $v=0$ and the equilibrium points at $a=0$.

Table 4. Inclination angle, Coulomb friction coefficient and limit velocity for different glider masses.

\begin{tabular}{lccc}
\hline & $\alpha\left[^{\circ}\right]$ & $\mu$ & $v_{\text {lim }}[\mathrm{m} / \mathrm{s}]$ \\
\hline $\mathrm{m} 1: 335 \mathrm{~g}$ & 4.3110 & 0.0071 & 0.2420 \\
$\mathrm{~m} 2: 435 \mathrm{~g}$ & 4.2944 & 0.0048 & 0.3232 \\
$\mathrm{~m} 3: 535 \mathrm{~g}$ & 4.2980 & 0.0040 & 0.3988 \\
\hline
\end{tabular}

The $a-v$ diagram in figure 9 shows that the system has a stable dynamical equilibrium point with positive limit velocity that grows with the glider mass. The values of $v_{\text {lim }}$ shown in Table 4 have been obtained as the $v$ intercept of the fit of the interval of positive velocities.

It is interesting to point out to the students that, using two independent movements, where the glider is launched with different velocities and even in different directions, the system evolves towards the same stable dynamical state with the same limit velocity. 


\section{Discussion}

In this work we present an experiment that illustrates the application of the phase diagrams to the study of a dynamical system with viscous friction. We have utilized a mechanical system with magnetic damping by Eddy currents. This setup produces linear friction [21, 24] avoiding the turbulences that appear when using fluids. Moreover it allows adjusting quite well the desired degree of friction, facilitating the work at low velocities. This aspect may be critical when using video analysis, because of the limitation in frame rate.

In order to obtain the phase diagrams, we have recorded the movement with video analysis obtaining $x(t)$, which has been numerically differentiated to compute $v(t)$ and $a(t)$, without imposing any predefined model to the data. A linear fit of these results produces a function $a(v)$ that is represented with the experimental data.

The use of this kind of graphics allows analyzing the movement evolution without having to integrate differential equations. In the case of glider with magnetic damping, the expected model is a linear relation $a(v)$, which is in good agreement with the experimental data. Moreover, it is shown that the intersection of the line $a(v)$ with the axis $a=0$ gives the limit velocity, corresponding to the dynamical equilibrium point. This point has been obtained for different masses, verifying that, for the same configuration of the track, the limit velocity increases with the mass.

The determination of the limit velocity for each mass has been performed with different initial conditions $\left(v_{0}<v_{\text {lim }}\right.$ and $\left.v_{0}>v_{\text {lim }}\right)$. Nevertheless, for any configuration, the fit can be made with all the data, offering a precise estimation of $v_{\text {lim }}$.

Moreover, we have analyzed the discontinuity that appears when the movement changes the direction $(v=0)$. This discontinuity does not respond to the viscous friction and is explained by the existence of a small Coulomb friction.

The present analysis has a high formative value for the students, which have the preconception of associating Coulomb friction to constant acceleration. By analyzing the movement dynamics with the phase diagram, it is obvious that the Coulomb friction has a mathematical behavior more complex than expected. Indeed, it is not a constant force, but it introduces a discontinuity in the acceleration, which can make very complex the analytical solution of the problems with Coulomb friction. Only in the particular cases where the movement does not change its direction, one can assume that the force is constant.

The utilization of phase diagrams in the teaching of first year mechanics is not habitual. Although, it can offer a complementary vision to the traditional experiments, which are usually based on the experimental measurement of the movement, $x(t)$ and the comparison of these results with the formulas obtained by integrating the movement equations associated to a 
particular dynamical model. In our opinion, this traditional approach limits quite the scope of the experiments.

On one hand, it implies that the students know how to integrate the differential equation of the movement and how to perform the nonlinear fits corresponding to expressions involving exponential and hyperbolic functions.

On the other hand, the direct comparison between the measured functions $x(t)$ and the functions of the model can be few sensitive to the discrepancies between them. As it has been proved in [11], important changes in the differential equations can result in small modifications in the solution $x(t)$. For example, in our case, the effect of Coulomb friction could be explained by a slight variation of the slope in the fitting results. In the same way, it is difficult to know from a fit of $x(t)$ if the movement is produced by a friction linear, quadratic or of another order.

In contrast, the phase diagrams allow working directly with the differential equation of the movement. Thereby, the students' attention is focused on the comprehension of the relations between the movement variables that define the system dynamics instead of the resolution of a mathematical problem. In our case, we check the linearity of the force of magnetic damping, analyze the meaning of limit velocity and verify that it depends not only on the friction, but also on the mass.

The main difficulty of the procedure is the need for determining with precision the velocity and the acceleration, which requires having a video camera with enough frame rate and using efficient methods of numerical differentiation.

Our experiment has been recorded at $200 \mathrm{fps}$, frequency quite higher than that of the conventional cameras. Nevertheless, there are affordable industrial cameras working at these or even higher frequencies. Anyway, it is not necessary that each student has a photogrammetry device, because the videos can be recorded in a unique device and then individually used for their processing.

Regarding to the smoothing and numerical differentiation algorithm, it is a computing procedure not difficult to explain at qualitative level and that can be understood as a black box that receives an input, the raw data, and produces an output, the smoothed data end their two first derivatives. In fact, many video analysis systems embody some smoothing and differentiation procedure that could be used as long as the frame rate is high enough.

\section{Conclusions}

The phase diagrams can be a useful tool in the mechanics teaching of first-year university. On one hand, they allow interpreting the dynamical behavior of a system without having to solve complex equations. On the other hand, they are a very sensitive method for checking the validity of the methods, because they allow checking the relations between the movement 
variables and not only the position function, as in the usual experiments. Applied to systems with friction, they ease the comprehension of the limit velocity as dynamical equilibrium point and allow analyzing the differences between different kinds of friction.

\section{References}

[1] Larson R F 1998 Measuring the coefficient of friction of a low-friction cart Phys. Teach. 36 464-465

[2] Paetkau M, Bahniwal M \& Gamblen J 2008 Magnetic Low-Friction Track. Phys. Teach. 46 307-309

[3] Takahashi K, Thompson D 1999 Measuring air resistance in a computerized laboratory. Am. J. Phys. 67 709-11

[4] Calderon S E, López González S, Gil S 2007 Determinación de la fuerza de roce con el aire usando nuevas tecnologías 2007 Rev. de Ens. de la Física 20 55-66

[5] Lindemuth J 2005 The effect of air resistance on falling balls. Am. J. Phys., 39 757-759

[6] Andereck B S 1999 Measurement of air resistance on an air track. Am. J. Phys., 67 528-533

[7] Pantaleone J and Messer J 2011 The added mass of a spherical projectile Am. J. Phys. 79 $1202-1210$

[8] Feinberg G 1965 Fall of bodies near the earth. Am. J. Phys. 33501

[9] Bohren C F 2004 Dimensional analysis, falling bodies, and the fine art of not solving differential equations Am. J. Phys. 72 534-7

[10] Munson B, Young D and Okiishi T 1994 Fundamentals of Fluid Mechanics (New York: Wiley)

[11] Moreno R, Page A, Riera J and Hueso J L 2014 Experimental analysis of nonlinear oscillations in the undergraduate physics laboratory Eur. J. Phys. 35015005.

[12] Erlichson H 1983 Maximum projectile range with drag and lift, with particular application to golf Am. J. Phys. 51 357-362

[13] Brancazio P. J. 1985 Looking into Chapman's homer: The physics of judging a fly ball Am. J. Phys. 53 849-855

[14] Parker G W 1977 Projectile motion with air resistance quadratic in the speed Am. J. Phys. 45 606-10

[15] Page A, Candelas P and Belmar F 2006 On the use of local fitting techniques for the analysis of physical dynamic systems Eur. J. Phys. 27 273-9

[16] Shone R 2002 Economic Dynamics: Phase diagrams and their economic application (Cambridge: Cambridge University Press)

[17] Murray J D 2002 Mathematical Biology I: An Introduction (Interdisciplinary Applied Mathematics vol. 17) ed S S Antman et al (New York: Springer)

[18] Van Buskirk R, Jeffries C 1985 Observation of chaotic dynamics of coupled nonlinear oscillators. Phys. Rev. A 31 3332-57.

[19] Siahmakoun A, French V A and Patterson J 1997 Nonlinear dynamics of a sinusoidally driven pendulum in a repulsive magnetic field Am. J. Phys. 65 393-400.

[20] Jordan D W and Smith P 1999 Nonlinear Ordinary Differential Equations: An Introduction to Dynamical Systems (Oxford: Oxford University Press)

[21] Vidaurre A, Riera J, Monsoriu J A, and Giménez M H, Testing theoretical models of magnetic damping using an air track, Eur. J. Phys. 29, 335-43

[22] González Hernández A and Mora C 2010 Freno Magnético Lat. Am. J. Phys. Educ. 4 92833. 
[23] Page A, Moreno R, Candelas P, and Belmar F. 2008 The accuracy of webcams in 2d motion analysis: sources of error and their control. Eur. J. Phys. 29 857-870.

[24] Cadwell L H. 1996 Magnetic damping: analysis of an Eddy current brake using an airtrack Am. J. Phys. 64 917-23 